\title{
An objective nodal staging system for breast cancer patients undergoing neoadjuvant systemic treatment
}

\author{
Tae-Kyung Yoo ${ }^{1,2,5+}$, Jung Min Chang ${ }^{3 \dagger}$, Hee-Chul Shin ${ }^{4}$, Wonshik Han ${ }^{1,2}$, Dong-Young Noh ${ }^{1,2}$
} and Hyeong-Gon Moon ${ }^{1,2^{*}}$ (D)

\begin{abstract}
Background: In this study, we aimed to develop an objective staging system to determine the degree of nodal metastasis in breast cancer patients undergoing neoadjuvant systemic treatment (NST).

Methods: We reviewed the pretreatment computed tomography (CT) images of 392 breast cancer patients who received NST. The association between the patterns of the enlarged regional lymph nodes and treatment outcome was analyzed.

Results: In the development cohort of 260 patients, 88 (33.8\%) patients experienced tumor recurrence and had a significantly higher number of enlarged lymph nodes on the pretreatment $\mathrm{CT}$ compared to patients with no recurrence. When patients were classified according to the numbers and locations of enlarged lymph nodes on pretreatment $\mathrm{CT}$, the number of lymph nodes larger than $1 \mathrm{~cm}$ was most significantly associated with tumor recurrence. The accuracy of the CT-based nodal staging system was validated in an independent cohort of 132 patients. The presence of the enlarged supraclavicular nodes was associated with worse outcome, but the effect seemed to originate from the accompanied extensive axillary nodal burden. The prognostic effect of the objectively measured axillary nodal metastasis was more pronounced in hormone receptor-negative tumors.

Conclusions: We have developed and validated an objective method of nodal staging in breast cancer patients who undergo NST based on the number of enlarged axillary lymph nodes. Our system can improve the current subjective approach, which uses physical examination alone.
\end{abstract}

Keywords: Breast cancer, Neoadjuvant systemic therapy, Nodal staging, Chest CT

\section{Background}

Neoadjuvant systemic therapy (NST) is increasingly used for the treatment of operable breast cancer in patients [1]. NST has been shown to increase the rate of breast conservation without compromising survival $[2,3]$. The increased use of NST has given rise to some controversial issues such as the optimal method of determining the residual extent of tumor and the use of sentinel node

\footnotetext{
*Correspondence: moonhg74@snu.ac.kr

${ }^{\dagger}$ Equal contributors

'Department of Surgery, Seoul National University College of Medicine, 03080, 101 Daehak-ro, Jongno-gu, Seoul, Republic of Korea

${ }^{2}$ Laboratory of Breast Cancer Biology, Cancer Research Institute, Seoul National University College of Medicine, 103 Daehak-ro, Jongno-gu, Seoul, Republic of Korea

Full list of author information is available at the end of the article
}

biopsy after NST [4-6]. In this study, we raise another clinically important issue for breast cancer patients who receive NST: the issue of initial axillary staging. The decision for post-NST axillary management and adjuvant radiation therapy often relies on the initial axillary nodal status. As more patients with early breast cancer receive NST, the importance of accurate initial axillary nodal staging is increasing.

In breast cancer patients who undergo primary surgery, the number of metastatic lymph nodes is a major prognostic factor, and the risk of recurrence is proportional to the degree of disease burden in the axillary lymph nodes $[7,8]$. In contrast, it is often difficult to obtain an accurate estimation of the extent of nodal involvement in patients who undergo NST. The current 
TNM staging system recommends physical examination to determine the N stage in patients receiving NST, based on the presence of the fixed nature or locations of the palpable nodes [9]. Unfortunately, studies have reported limited accuracy of physical examination in determining clinical $\mathrm{N}$ stage in patients with breast cancer, with a sensitivity around 30\% [10-13]. Furthermore, clinical N staging with physical examination is a highly subjective method, and the accuracy may vary among surgeons.

In this study, we aimed to develop an objective staging system based on initial computed tomography (CT) images that can provide improved prognostic information for patients who receive NST.

\section{Methods}

The medical records of patients who underwent NST and surgery for invasive breast cancer at Seoul National University Hospital (SNUH) between Jan 2006 and Dec 2011 were reviewed retrospectively. In our institution, patients who undergo neoadjuvant systemic therapy often received chest $\mathrm{CT}$ to rule out visceral metastasis and to assess the degree of nodal enlargement prior to the administration of the systemic treatment. For the present study, we excluded the patients who underwent palliative surgery or had a history of breast cancer. Also, patients who did not have chest CT images at diagnosis or who had poor-quality images were excluded. Patients were divided into two cohorts, in the ratio of two to one, for development and validation of a CT-based nodal staging system (development cohort and validation cohort, respectively). Patients' data including clinicopathologic, treatment-related, and survival information were obtained from SNUH Breast Cancer Center database, which is a prospectively maintained web-based database [14]. In all individuals, CT scanning was performed at end-inspiration following hyperventilation. CT imaging was performed using the following scanners: Genesis Hispeed and LightSpeed Ultra; GE Healthcare, Milwaukee, WI, USA; Somatom Plus-4 and Sensation-16; Siemens Medical Systems, Erlangen, Germany; Brilliance-64; Phillips Medical Systems, the Netherlands. Two boardcertified radiologists (mean 13 years of experience) who were blind to the clinical staging and treatment outcome information evaluated the CT scans independently. On the CT scans, lymph node status was evaluated based on the size and location (axillary levels I, II, and III, supraclavicular lymph node (SCN) or internal mammary lymph node (IMN)). At each location, the number of lymph nodes larger than $1 \mathrm{~cm}$ and larger than $2 \mathrm{~cm}$ was separately recorded. Discordant data from two radiologists were reevaluated by the same two radiologists to reach a consensus, and the final integrated results were recorded.
All patients were treated with anthracycline- and/or taxane-based chemotherapy regimens. Among the patients who had a human epidermal growth factor receptor 2 (HER2)-amplified tumor, 15\% (17/113) received a HER2-related targeted therapy preoperatively. All patients received postoperative hormonal or radiation therapy, if indicated. Postoperative follow-up with the clinic was done at least every 6 months and included a routine physical examination, laboratory testing, breast ultrasonography, mammography and chest $\mathrm{X}$-ray. Additional examinations were done at the physician's discretion.

In this study, we used two additional groups of patients to assess the negative predictive value of $\mathrm{CT}$ evaluation and to estimate the hormone receptor (HRc)dependent prognostic impact of nodal status. To assess the negative predictive value of the axillary nodal status as evaluated by chest $\mathrm{CT}$, the medical records of breast cancer patients who received primary surgery as their initial treatment between January 2014 and December 2014 were reviewed for CT findings and pathological N staging (supplementary cohort A). To demonstrate the HRc-dependent prognostic effect, we selected consecutive patients who received primary surgery between July 2005 and June 2008 in whom adequate survival information was available (supplementary cohort B).

Univariate analysis, using the Pearson chi-square test, was performed to compare clinicopathologic features of the development and validation cohorts. Univariate survival analysis for development and validation of the CT-based nodal staging system was performed using Kaplan-Meier survival analysis and log-rank tests. Multivariate survival analysis was conducted using a Cox proportional hazards regression model. Prognostic factors with statistical significance in the univariate analysis were entered in the Cox regression model. Disease-free survival (DFS) was defined as the time from start of neoadjuvant systemic therapy to the date of breast cancer recurrence, death from any cause or final outpatient clinic visit. Breast cancer recurrence was defined as locoregional recurrence or distant metastasis, and contralateral breast recurrences were excluded.

\section{Results}

Patient characteristics and the prevalence of CT-detected lymph node enlargement

We reviewed the data of 536 breast cancer patients who received NST between January 2006 and December 2011. The median follow-up period was 63 months (range, 2-118 months). Among them 68 (12.7\%) patients had metastatic disease at diagnosis, $1(0.2 \%)$ patient had palliative surgery due to chest wall invasion and $2(0.4 \%)$ patients had a history of breast cancer and were excluded. Also, 49 (9.1\%) patients had no pre-NST chest 
CT scan, and 24 (4.5) patients had poor-quality images. After exclusion, a total of 392 patients were included in this study. The clinical and pathologic characteristics of the included patients are shown in Table 1. Clinically, 282 patients $(71.9 \%)$ had stage III breast cancer.
The nodal status of each patient was assessed using chest CT images obtained before the initiation of the systemic chemotherapy. Among the 392 patients, 69 patients (17.6\%) showed no enlarged lymph nodes in the axillary, supraclavicular, or internal mammary nodal

Table 1 Clinical characteristics of the patients

\begin{tabular}{|c|c|c|c|c|}
\hline & & Development n (\%) & Validation n (\%) & $p$ \\
\hline & & $(n=260)$ & $(n=132)$ & \\
\hline Age (median, range) & & $46(24-78)$ & $46(27-72)$ & \\
\hline \multirow[t]{2}{*}{ Breast Surgery } & Breast conserving surgery & $120(46.2)$ & $63(47.7)$ & 0.768 \\
\hline & Total mastectomy & $140(53.8)$ & $69(52.3)$ & \\
\hline \multirow[t]{2}{*}{ Axilla Surgery } & Sentinel lymph node biopsy ${ }^{a}$ & $13(5.0)$ & $8(6.2)$ & 0.634 \\
\hline & Axillary lymph node dissection & $247(95.0)$ & $122(93.8)$ & \\
\hline \multirow[t]{4}{*}{ Clinical T stage } & $\mathrm{T} 1$ & $3(1.2)$ & $4(3.0)$ & 0.061 \\
\hline & $\mathrm{T} 2$ & $126(48.5)$ & $54(40.9)$ & \\
\hline & T3 & $86(33.1)$ & $58(43.9)$ & \\
\hline & $\mathrm{T} 4$ & $45(17.3)$ & $16(12.1)$ & \\
\hline \multirow[t]{2}{*}{ AJCC Stage } & $\|$ & $75(28.8)$ & $35(26.5)$ & 0.627 \\
\hline & III & $185(71.2)$ & $97(73.5)$ & \\
\hline \multirow[t]{3}{*}{ Histology } & Ductal & $237(91.2)$ & $121(91.7)$ & 0.528 \\
\hline & Lobular & $6(2.3)$ & $5(3.8)$ & \\
\hline & Mixed/other & $17(6.5)$ & $6(4.5)$ & \\
\hline \multirow[t]{3}{*}{ Grade } & Low (I and II) & $105(40.4)$ & $58(43.9)$ & 0.667 \\
\hline & High (III) & $127(48.8)$ & $63(47.7)$ & \\
\hline & Unknown & $28(10.8)$ & $11(8.3)$ & \\
\hline \multirow[t]{2}{*}{ HRc status } & Positive & $164(63.1)$ & $95(72.0)$ & 0.079 \\
\hline & Negative & $96(36.9)$ & $37(28.0)$ & \\
\hline \multirow[t]{3}{*}{ HER2 status } & Positive & $66(25.4)$ & $46(34.8)$ & 0.072 \\
\hline & Negative & $193(74.2)$ & $86(65.2)$ & \\
\hline & Unknown & $1(0.4)$ & $0(0.0)$ & \\
\hline \multirow[t]{5}{*}{ Subtype } & $\mathrm{HRc}+/ \mathrm{HER} 2-$ & $131(50.4)$ & $69(52.3)$ & 0.137 \\
\hline & HRc+/HER2+ & $33(12.7)$ & $26(19.7)$ & \\
\hline & HRc-/HER2+ & $34(13.1)$ & 18 (13.6) & \\
\hline & HRc-/HER2- & $61(23.5)$ & 19 (14.4) & \\
\hline & Unknown & $1(0.4)$ & $0(0.0)$ & \\
\hline \multirow[t]{3}{*}{ Ki-67 } & $<10 \%$ & $133(51.2)$ & $72(54.5)$ & 0.705 \\
\hline & $\geq 10 \%$ & $121(46.5)$ & $56(42.4)$ & \\
\hline & Unknown & $6(2.3)$ & $4(3.0)$ & \\
\hline \multirow[t]{4}{*}{ Type of NST } & Anthracyclines & $8(3.1)$ & $6(4.5)$ & 0.879 \\
\hline & Anthracyclines and Taxanes & 239 (91.9) & $120(90.9)$ & \\
\hline & Taxanes & $10(3.8)$ & $5(3.8)$ & \\
\hline & Others & $3(1.2)$ & $1(0.8)$ & \\
\hline \multirow[t]{3}{*}{ Anti-HER2 Therapy } & Neoadjuvant & $12(4.6)$ & $6(4.5)$ & 0.968 \\
\hline & Adjuvant & $52(19.9)$ & $25(18.9)$ & \\
\hline & No & $196(75.5)$ & $101(76.5)$ & \\
\hline
\end{tabular}


chains. We evaluated the lymph node status based on size thresholds $(1 \mathrm{~cm}$ or $2 \mathrm{~cm}$ ) and locations (axillary levels I, II, or III, and SCN, or IMN) of the enlarged nodes (Fig. 1).

To estimate the possibility of axillary lymph node involvement in patients who had no visible lymph node enlargement on CT, we analyzed the incidence of lymph node metastasis in 605 early breast cancer patients who underwent chest CT before primary surgery between January 2014 and December 2014 (supplementary cohort A). In this supplementary cohort, all patients initially underwent sentinel lymph node biopsy and only proceeded to axillary lymph node dissection when intraoperative frozen section biopsy identified lymph node involvement. The incidence of lymph node involvement was $17.7 \%$, and most patients had N1 stage disease (Additional file 1: Table S1).

\section{Development and validation of the CT-based nodal staging system}

Patients were randomly assigned to the development or validation cohort. The incidence of known prognostic factors did not differ between the development and validation cohorts (Table 1). In the development cohort of 260 patients, we first analyzed the factors associated with tumor recurrence. In this cohort, 88 patients experienced tumor recurrence during the follow-up period (Fig. 2a). As expected, the patients who experienced tumor recurrence had a significantly higher number of enlarged lymph nodes on the pretreatment CT (Fig. 2b). Cox regression analysis showed that an increase in the number of enlarged lymph nodes of more than $1 \mathrm{~cm}$ was associated with a $7.2 \%$ increased risk of recurrence (Table 2). After observing the prognostic significance, we compared various methods of nodal staging in predicting DFS. Patients were classified according to the number and location of the enlarged nodes. Among the various nodal classification methods, the accuracy of predicting recurrence was highest when the patients were classified according to the number of enlarged lymph nodes ( $>1$ $\mathrm{cm}$ diameter, Fig. 2c-e). Also, when comparing with clinical $\mathrm{N}$ staging by conventional methods, the concordance rate was very low (kappa value 0.086; 95\% CI 0.002-0.170; Additional file 1: Table S3), and the prognostic value of conventional methods was also inadequate (Additional file 1: Figure S1).

We analyzed the prognostic importance of the CTbased nodal staging system in an independent validation cohort of 132 patients who were treated with neoadjuvant systemic therapy during the same period. In the validation cohort, 29 breast cancer recurrence events occurred during the follow-up period. The staging system could effectively predict the survival outcome when patients were classified according to the number of enlarged nodes $>1 \mathrm{~cm}$ in size (Fig. 2f).

\section{Prognostic significance of extra-axillary lymph node enlargement}

Traditionally, patients with lymph node metastases in the extra-axillary area are expected to have worse outcomes compared to patients whose lymph node metastases are contained in the axillary area. We assessed the relationship between extra-axillary lymph node enlargement and survival outcome in the entire cohort of 392 patients.

Twenty-one (5.4\%) and twelve (3.1\%) patients had enlarged supraclavicular lymph nodes and internal mammary lymph nodes, respectively. The presence of internal mammary node enlargement was not associated with an increased risk of recurrence regardless of axillary nodal involvement (Fig. 3a). Patients with enlarged supraclavicular lymph nodes showed significantly shorter DFS, but the prognostic significance was lost in patients with more than three enlarged axillary lymph nodes (Fig. 3b). Eighteen patients (85.7\%) with enlarged supraclavicular lymph nodes also had more than three enlarged lymph nodes. Our data suggest that the prognostic significance of enlarged supraclavicular lymph nodes was mostly derived from the accompanied axillary nodal involvement.

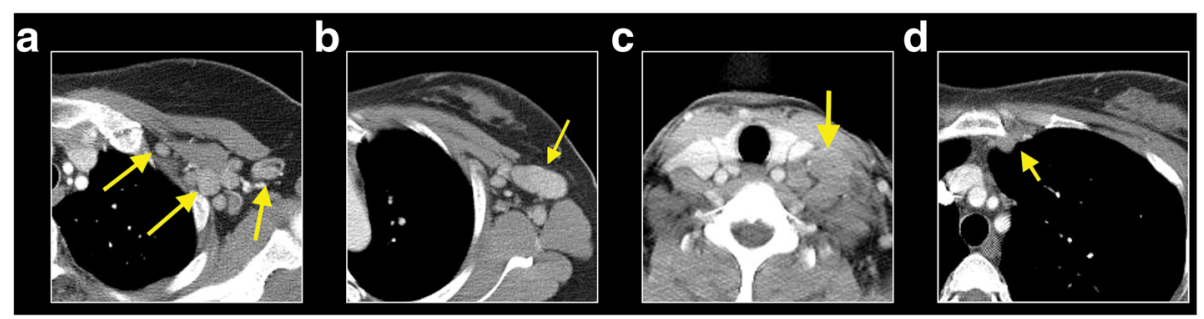

Fig. 1 The representative CT images of the regional lymph node enlargements.Yellow arrows indicate the presence of the enlarged lymph nodes in axillary level I-III (a), a lymph node larger than $2 \mathrm{~cm}$ in level I (b), enlarged supraclavicular lymph node (c), and an internal mammary node (d) 


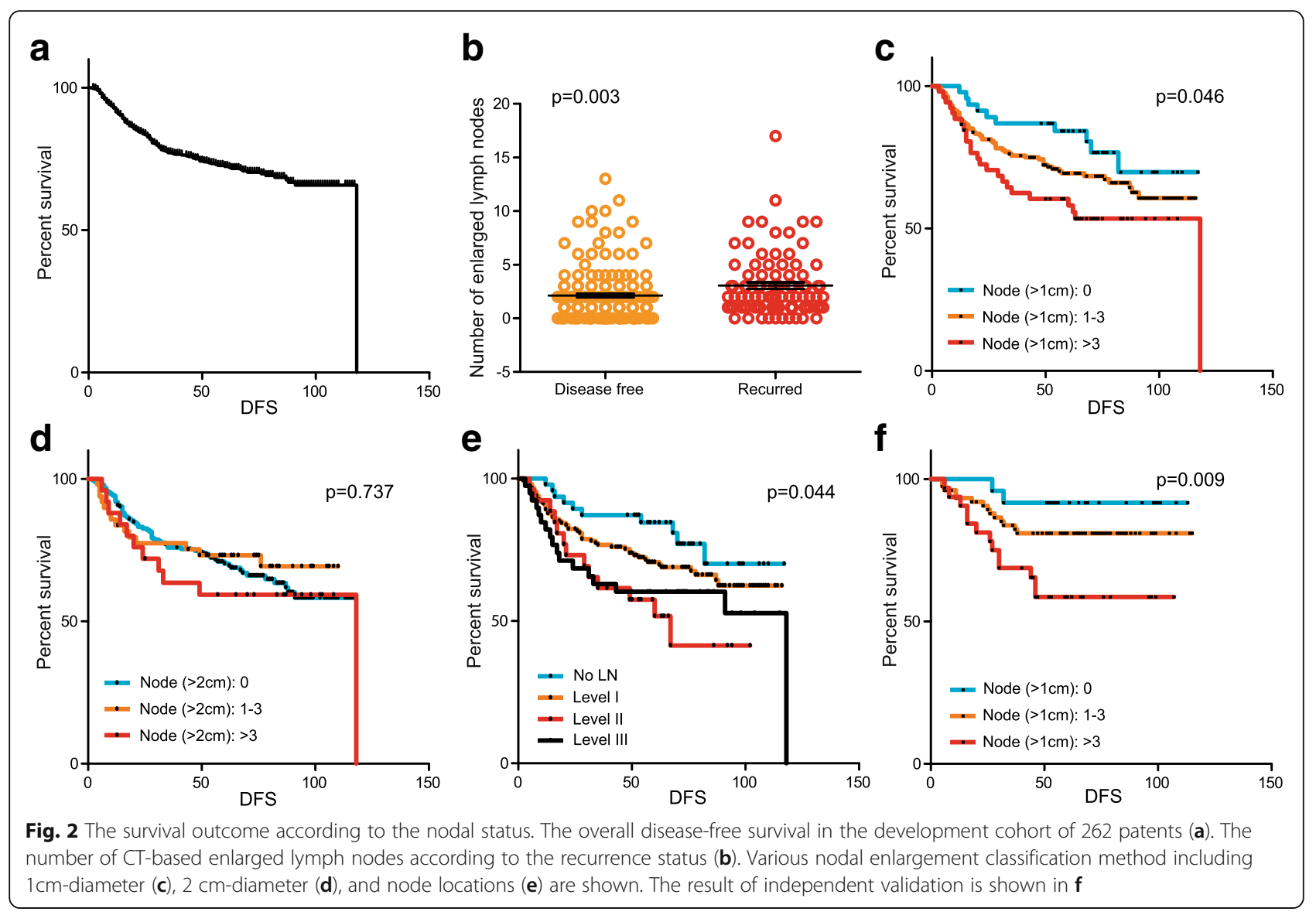

\section{Nodal involvement and hormone receptor status}

The prognostic significance of the CT-based nodal staging system was examined in both HRc-positive and HRc-negative patients in the entire cohort. The staging system more clearly predicted the treatment outcomes of patients with HRc-negative tumors (Fig. 4a). To assess whether this HRc-dependent prognostic effect was a limitation of the present CT-based staging system or a result of the intrinsic molecular characteristics of the HRc-positive tumors, we analyzed the prognostic

Table 2 Univariate and multivariate analysis of prognostic factors in the development cohort $(n=260)$

\begin{tabular}{|c|c|c|c|c|c|c|c|c|}
\hline \multirow[b]{3}{*}{ Age } & \multicolumn{4}{|c|}{ Univariate } & \multicolumn{4}{|c|}{ Multivariate } \\
\hline & \multirow{2}{*}{$\begin{array}{l}P \\
0.643\end{array}$} & \multirow{2}{*}{$\frac{H R}{0.995}$} & \multicolumn{2}{|c|}{$95 \% \mathrm{Cl}$ for $\mathrm{HR}$} & \multirow[t]{2}{*}{$P$} & \multirow[t]{2}{*}{$\mathrm{HR}$} & \multicolumn{2}{|c|}{$95 \% \mathrm{Cl}$ for HR } \\
\hline & & & 0.973 & 1.017 & & & & \\
\hline Tumor size & 0.027 & 1.094 & 1.010 & 1.184 & 0.176 & 1.059 & 0.975 & 1.151 \\
\hline Axillary LN (1 cm) & 0.006 & 1.097 & 1.028 & 1.172 & 0.051 & 1.072 & 1.000 & 1.149 \\
\hline High HG & 0.001 & 2.226 & 1.377 & 3.598 & 0.014 & 1.934 & 1.146 & 3.266 \\
\hline HRc negative & 0.005 & 1.841 & 1.204 & 2.815 & & & & \\
\hline HER2 positive & 0.608 & 1.132 & 0.706 & 1.814 & & & & \\
\hline Subtype $^{a}$ & 0.072 & & & & 0.354 & & & \\
\hline HRc+/HER2+ & 0.493 & 0.765 & 0.357 & 1.642 & 0.228 & 0.617 & 0.282 & 1.352 \\
\hline HRc-/HER2+ & 0.043 & 1.857 & 1.050 & 3.381 & 0.226 & 1.469 & 0.788 & 2.737 \\
\hline HRc-/HER2- & 0.036 & 1.717 & 1.035 & 2.847 & 0.468 & 1.222 & 0.711 & 2.103 \\
\hline Ki67 $\geq 10 \%$ & 0.322 & 1.240 & 0.810 & 1.898 & & & & \\
\hline
\end{tabular}

$P$ values are derived from univariate or multivariate Cox proportional hazard models

$H R$ hazard ratio; $\mathrm{Cl}$ confidence interval; $L N$ lymph node; $H G$ histologic grade; $H R c$ hormone receptor; $H E R 2$ human epidermal growth factor receptor 2

${ }^{\mathrm{a}} \mathrm{HRC}+/$ HER2- cases were used as reference group 


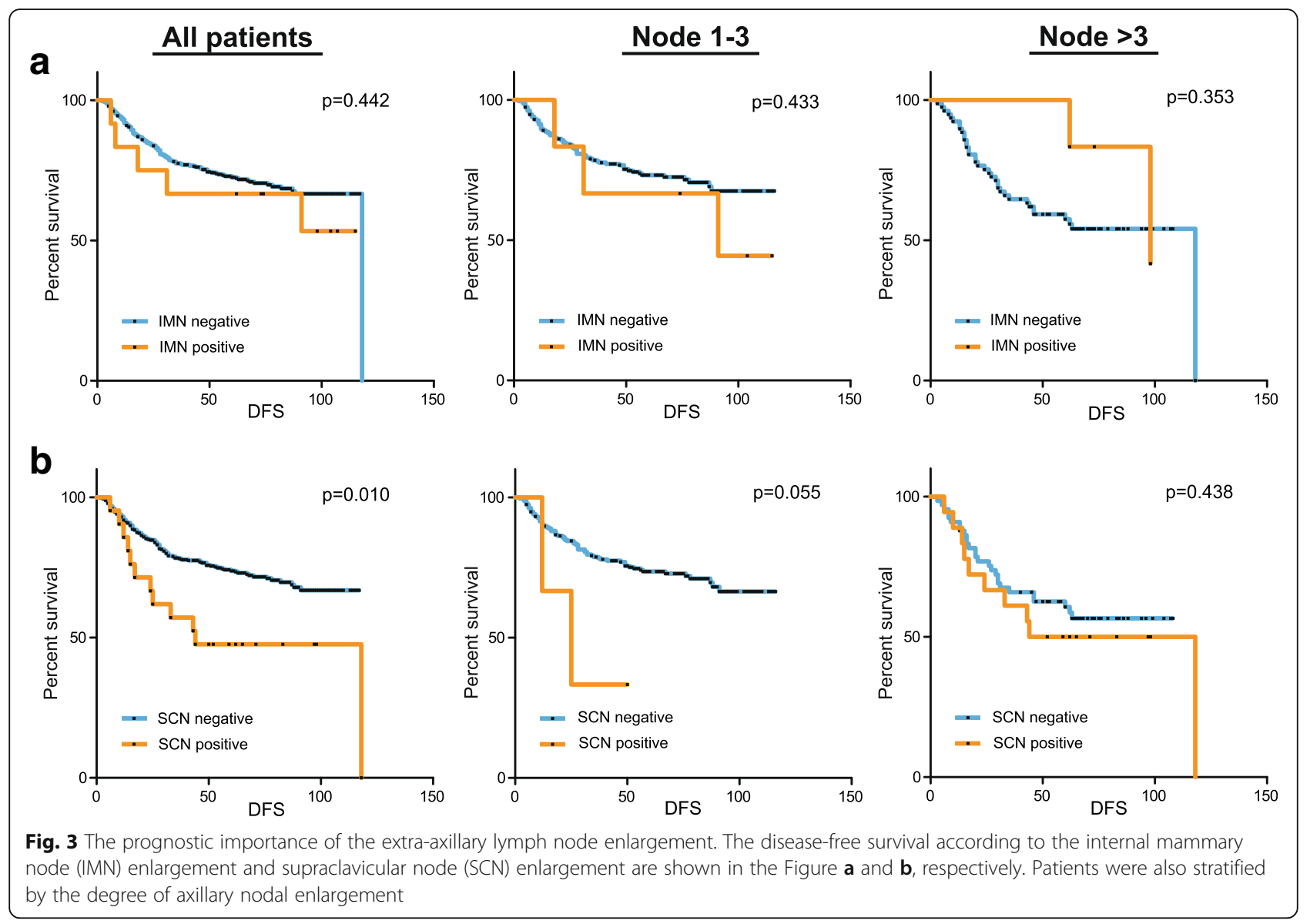

significance of the pathologic $\mathrm{N}$ stage in 1702 breast cancer patients who underwent primary surgery between July 2005 and June 2008 at our institution (supplementary cohort B). The basic clinicopathologic characteristics of these patients are described in Additional file 1: Table S2. The relationship between the risk of recurrence and the pathologic $\mathrm{N}$ stages in these patients showed similar trends according to HRc status (Fig. 4b) suggesting that the HRc- dependent prognostic implications reflect the biologic characteristics of the breast cancer.

\section{Discussion}

In the present study, we developed and validated an objective clinical $\mathrm{N}$ staging method using initial chest $\mathrm{CT}$ images of patients undergoing NST. When the patients were classified according to the number of axillary lymph a CT-based N staging in patients undergoing NST

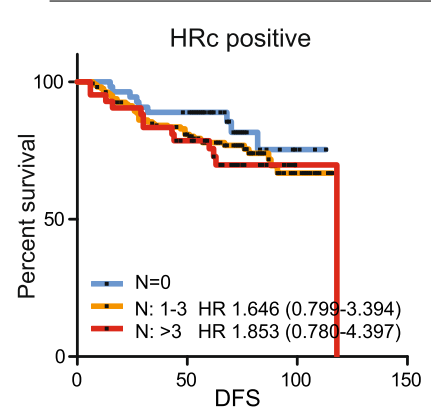

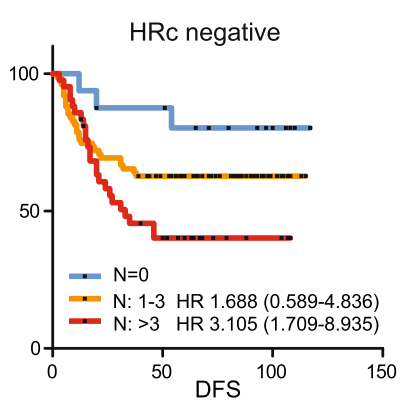

b

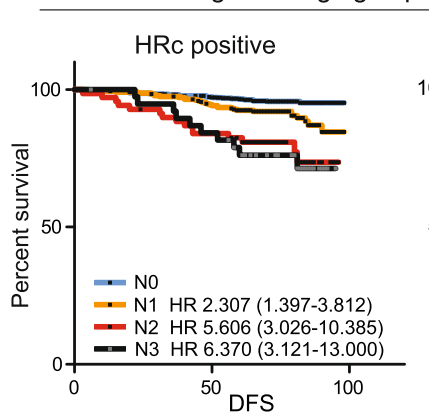

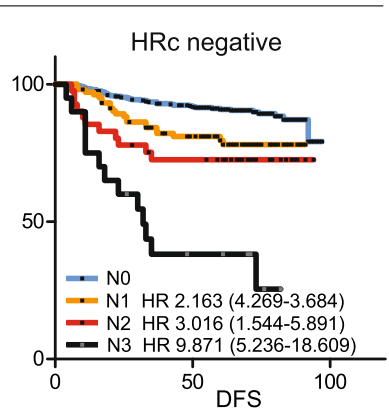

Fig. 4 Prognostic significance of the CT-based nodal staging system and the pathologic $N$ stages according to the hormonal receptor status. The prognostic significance of the CT-based nodal staging system in patients undergoing neoadjuvant systemic therapy (a) and the significance of the pathologic $N$ stages in patients undergoing primary surgery $(\mathbf{b})$ 
nodes larger than $1 \mathrm{~cm}$, we observed a significant and proportional increase in risk of recurrence. Our data suggest that a CT-based objective axillary staging using the number of enlarged nodes can be a useful alternative to conventional axillary staging done by a physical examination.

Many previous studies have attempted to explore the value of imaging studies, including contrast-enhanced breast magnetic resonance imaging, ultrasonography, and positron emission tomography (PET) scan, in determining nodal status in patients with breast cancer [15]. However, most studies have focused on the role of imaging studies in predicting the presence of nodal metastasis rather than evaluating the quantitative burden of nodal disease. Axillary ultrasound is an imaging modality associated with low cost and risk and is also reported to have high sensitivity in excluding or predicting heavy nodal burden [16-18]. However, quantification of nodal disease using ultrasound is difficult and operator dependent, which compromises the objectivity of the imaging test. The practicality of a PET-CT scan as a nodal staging modality has also been demonstrated in many studies recently [19-24]. For patients undergoing NST, Koolen et al. have demonstrated the role of PET-CT scan for quantification of axillary nodal status and evaluation of extra-axillary nodal involvement [19, 21]. A PET-CT scan performs with high sensitivity and specificity, but its lack of a standard cutoff level for standardized uptake values (SUVmax) and its dependency on the SUVmax of the main tumor are obstacles for objective axillary nodal classification [20, 21, 24]. Moreover, considering the cost and availability of this technique, PET-CT may be best reserved as an adjunct for indeterminate lesions [25]. Compared to these imaging modalities, axillary staging done by chest $\mathrm{CT}$ imaging has the advantages of a fairly low cost and high availability. Also, the staging system developed in this study enables physicians to quantify axillary nodal status through an objective and reproducible method for women undergoing NST.

It has been reported that extra-axillary lymph node metastasis, such as SCN and IMN, is associated with poor outcome in patients with breast cancer [9, 26-29]. In our study, we could not demonstrate an independent prognostic value of extra-axillary lymph node enlargement. While patients with enlarged SCN showed worse outcomes, they often had more than three enlarged axillary nodes. In patients with more than three enlarged axillary nodes, the presence of SCN did not confer a significant prognostic difference. Our data suggest that the known prognostic importance of extra-axillary nodal involvement can be the consequence of the degree of axillary metastatic burden rather than an independent prognostic factor. Indeed, Olivotto et al. [30] showed similar overall survival between patients with SCN metastasis and stage IIIB tumors, and Chen et al. [31] showed comparable outcomes between patients with $\mathrm{SCN}$ metastasis and N3 stage tumors.

The ability of our staging system to classify patients according to their risk of recurrence was more pronounced in HRc-negative tumors. This phenomenon was also seen in the pathologic nodal staging system for 1702 primary breast cancer patients who received surgery as their initial treatment (Supplementary cohort B). This can be explained by the effect of a higher baseline risk of recurrence for HRcnegative tumors compared to that of HRc-positive tumors, despite a similar increase in the relative risk [32, 33]. On the other hand, the prognostic impact of the degree of nodal involvement may differ according to the molecular characteristics of the breast cancer [34]. The relationship between nodal metastatic burden and the risk of recurrence according to the molecular subtypes of breast cancer should be examined further with a larger dataset.

The retrospective nature of this study is a major limitation. Various CT systems were used for evaluation of enlarged lymph nodes, causing minor differences in slice thickness, resolution, and image quality. A prospective validation study with a standardized protocol is needed to strengthen the value of our nodal staging system. Also, our patient cohort was mainly composed of stage III breast cancer patients (71.2\%); therefore, applying our results to early breast cancer patients may have some limitations. Other limitations include the lack of pathologic confirmation of extra-axillary node involvement and the lack of subtype-specific analysis due to the limited number of patients.

\section{Conclusion}

We have developed an objective nodal staging system for patients undergoing NST using the number of enlarged nodes on initial CT images. Our staging system can provide objective and reproducible prognostic information that can overcome the limitations of the current clinical staging system, which relies on the subjective findings of physical examination.

\section{Additional file}

Additional file 1: Figure S1. The survival outcome according to conventional clinical N stage in the development cohort. Table S1. The incidence of axillary node involvement in patients with no suspicious nodes on CT (supplementary cohort A). Table S2. Clinicopathologic characteristics of patients who underwent primary surgery between July 2005 and June 2008 (supplementary cohort B). Table S3. Comparison of the CT-based nodal staging system and conventional clinical $\mathrm{N}$ staging (development cohort). (DOCX $83 \mathrm{~kb}$ )

\section{Abbreviations}

Cl: confidence interval; CT: computed tomography; DFS: disease-free survival; HER2: human epidermal growth factor receptor 2; HG: histologic grade; HR: hazard ratio; HRC: hormone receptor; IMN: internal mammary lymph 
node; LN: Iymph node; NST: neoadjuvant systemic therapy; PET: positron emission tomography; SCN: supraclavicular lymph node; SNUH: Seoul National University Hospital; SUVmax: standardized uptake values

\section{Acknowledgements}

None.

\section{Funding}

This work was supported by the Basic Science Research Program through the National Research Foundation of Korea (NRF) funded by the Ministry of Education, Science and Technology (NRF-2015R1D1A1A02061904) and by the grant from the National R\&D Program for Cancer Control, Ministry for Health and Welfare, Republic of Korea (A1520250).

The funding body had no role in the design of the study and collection, analysis and interpretation of the data and in writing of the manuscript.

\section{Availability of data and materials}

The datasets used and/or analyzed during the current study are available from the corresponding author on reasonable request.

\section{Authors' contributions}

TKY and HGM analyzed and interpreted patient data and were major contributors in writing the manuscript. JMC evaluated all imaging data and participated in the writing and revision of the imaging part of the manuscript. HCS, WH and DYN provided patient data, contributed in interpretation of the data and were involved in the revision of the manuscript. All authors read and approved the final manuscript.

\section{Competing interests}

The authors declare that they have no competing interests.

\section{Consent for publication}

Not applicable.

\section{Ethics approval and consent to participate}

This study was approved by the Institutional Review Board of Seoul National University Hospital (IRB No. 1512-004-723), which waived informed consent, and was conducted according to the principles expressed in the Declaration of Helsinki.

\section{Publisher's Note}

Springer Nature remains neutral with regard to jurisdictional claims in published maps and institutional affiliations.

\section{Author details}

'Department of Surgery, Seoul National University College of Medicine, 03080, 101 Daehak-ro, Jongno-gu, Seoul, Republic of Korea. 'Laboratory of Breast Cancer Biology, Cancer Research Institute, Seoul National University College of Medicine, 103 Daehak-ro, Jongno-gu, Seoul, Republic of Korea. ${ }^{3}$ Department of Radiology, Seoul National University College of Medicine, 101 Daehak-ro, Jongno-gu, Seoul, Republic of Korea. ${ }^{4}$ Department of Surgery, Chung-Ang University College of Medicine, 84 Heukseouk-ro, Dongjak-gu, Seoul, Republic of Korea. ${ }^{5}$ Present address: Department of Surgery, Seoul St. Mary's Hospital, College of Medicine, The Catholic University of Korea, 222 Banpo-daero, Seocho-gu, Seoul, Republic of Korea.

Received: 28 December 2016 Accepted: 22 May 2017

Published online: 31 May 2017

\section{References}

1. Graham PJ, Brar MS, Foster T, McCall M, Bouchard-Fortier A, Temple W, et al. Neoadjuvant chemotherapy for breast cancer, is practice changing? A population-based review of current surgical trends. Ann Surg Oncol. 2015;22:3376-82.

2. Mauri D, Pavlidis N, loannidis JP. Neoadjuvant versus adjuvant systemic treatment in breast cancer: a meta-analysis. J Natl Cancer Inst. 2005;97:188-94.

3. Mieog JS, van der Hage JA, van de Velde CJ. Neoadjuvant chemotherapy for operable breast cancer. Br J Surg. 2007;94:1189-200.

4. Kaufmann M, von Minckwitz G, Bear HD, Buzdar A, McGale P, Bonnefoi H, et al. Recommendations from an international expert panel on the use of neoadjuvant (primary) systemic treatment of operable breast cancer: new perspectives 2006. Ann Oncol. 2007;18:1927-34.

5. King TA, Morrow M. Surgical issues in patients with breast cancer receiving neoadjuvant chemotherapy. Nat Rev Clin Oncol. 2015;12:335-43.

6. Moon HG, Han W, Ahn SK, Cho N, Moon WK, Im SA, et al. Breast cancer molecular phenotype and the use of HER2-targeted agents influence the accuracy of breast MRI after neoadjuvant chemotherapy. Ann Surg. 2013:257:133-7.

7. Carter $\mathrm{CL}$, Allen $\mathrm{C}$, Henson DE. Relation of tumor size, lymph node status, and survival in 24,740 breast cancer cases. Cancer. 1989;63. $181-7$

8. Michaelson JS, Silverstein M, Sgroi D, Cheongsiatmoy SA, Taghian A, Powell S, et al. The effect of tumor size and lymph node status on breast carcinoma lethality. Cancer. 2003;98:2133-43.

9. Singletary SE, Allred C, Ashley P, Bassett LW, Berry D, Bland Kl, et al. Revision of the American joint committee on cancer staging system for breast cancer. J Clin Oncol. 2002;20:3628-36.

10. Valente SA, Levine GM, Silverstein MJ, Rayhanabad JA, Weng-Grumley JG, Ji $L$, et al. Accuracy of predicting axillary lymph node positivity by physical examination, mammography, ultrasonography, and magnetic resonance imaging. Ann Surg Oncol. 2012;19:1825-30.

11. Majid S, Tengrup I, Manjer J. Clinical assessment of axillary lymph nodes and tumor size in breast cancer compared with histopathological examination: a population-based analysis of 2,537 women. World J Surg. 2013;37:67-71.

12. Lanng C, Hoffmann J, Galatius H, Engel U. Assessment of clinical palpation of the axilla as a criterion for performing the sentinel node procedure in breast cancer. Eur J Surg Oncol. 2007;33:281-4.

13. Feng $Y$, Huang $R$, He $Y$, Lu A, Fan Z, Fan T, et al. Efficacy of physical examination, ultrasound, and ultrasound combined with fine-needle aspiration for axilla staging of primary breast cancer. Breast Cancer Res Treat. 2015:149:761-5

14. Moon HG, Han W, Noh DY. Underweight and breast cancer recurrence and death: a report from the Korean Breast Cancer Society. J Clin Oncol. 2009;27: 5899-905.

15. Bazan JG, White J. Imaging of the axilla before preoperative chemotherapy: implications for postmastectomy radiation. Cancer. 2015;121:1187-94.

16. Jackson RS, Mylander C, Rosman M, Andrade R, Sawyer K, Sanders T, et al. Normal axillary ultrasound excludes heavy nodal disease burden in patients with breast cancer. Ann Surg Oncol. 2015:22:3289-95.

17. Caudle AS, Kuerer HM, Le-Petross HT, Yang W, Yi M, Bedrosian I, et al. Predicting the extent of nodal disease in early-stage breast cancer. Ann Surg Oncol. 2014;21:3440-7.

18. Schipper RJ, van Roozendaal LM, de Vries B, Pijnappel RM, Beets-Tan RG, Lobbes MG, et al. Axillary ultrasound for preoperative nodal staging in breast cancer patients: is it of added value? Breast. 2013;22:1108-13.

19. Koolen $B B$, Valdes Olmos RA, Elkhuizen PH, Vogel W, Vrancken Peeters MJ, Rodenhuis S, et al. Locoregional lymph node involvement on 18F-FDG PET/ $\mathrm{CT}$ in breast cancer patients scheduled for neoadjuvant chemotherapy. Breast Cancer Res Treat. 2012;135:231-40.

20. An YS, Lee DH, Yoon JK, Lee SJ, Kim TH, Kang DK, et al. Diagnostic performance of 18F-FDG PET/CT, ultrasonography and MRI. Detection of axillary lymph node metastasis in breast cancer patients. Nuklearmedizin. 2014;53:89-94.

21. Koolen BB, Valdes Olmos RA, Vogel WV Vrancken Peeters MJ, Rodenhuis S, Rutgers EJ, et al. Pre-chemotherapy 18F-FDG PET/CT upstages nodal stage in stage II-III breast cancer patients treated with neoadjuvant chemotherapy. Breast Cancer Res Treat. 2013;141:249-54.

22. Robertson IJ, Hand F, Kell MR. FDG-PET/CT in the staging of local/regional metastases in breast cancer. Breast. 2011;20:491-4.

23. Ng SP, David S, Alamgeer M, Ganju V. Impact of pretreatment combined (18)F-Fluorodeoxyglucose positron emission tomography/ computed tomography staging on radiation therapy treatment decisions in locally advanced breast cancer. Int J Radiat Oncol Biol Phys. 2015;93:111-7.

24. Ahn JH, Son EJ, Kim JA, Youk JH, Kim EK, Kwak JY, et al. The role of ultrasonography and FDG-PET in axillary lymph node staging of breast cancer. Acta Radiol. 2010:51:859-65.

25. McCartan DP, Prichard RS, MacDermott RJ, Rothwell J, Geraghty J, Evoy D, et al. Role of bone scan in addition to $C T$ in patients with breast cancer selected for systemic staging. Br J Surg. 2016;103:839-44. 
26. Veronesi U, Cascinelli N, Bufalino R, Morabito A, Greco M, Galluzzo D, et al. Risk of internal mammary lymph node metastases and its relevance on prognosis of breast cancer patients. Ann Surg. 1983;198:681-4.

27. Cody HS 3rd, Urban JA. Internal mammary node status: a major prognosticator in axillary node-negative breast cancer. Ann Surg Oncol. 1995;2:32-7.

28. Jackson SM. Carcinoma of the breast-the significance of supraclavicular lymph node metastases. Clin Radiol. 1966;17:107-14.

29. Dellapasqua S, Bagnardi V, Balduzzi A, lorfida M, Rotmensz N, Santillo B, et al. Outcomes of patients with breast cancer who present with ipsilateral supraclavicular or internal mammary lymph node metastases. Clin Breast Cancer. 2014;14:53-60.

30. Olivotto IA, Chua B, Allan SJ, Speers CH, Chia S, Ragaz J. Long-term surviva of patients with supraclavicular metastases at diagnosis of breast cancer. J Clin Oncol. 2003:21:851-4.

31. Chen SC, Chang HK, Lin YC, Leung WM, Tsai CS, Cheung YC, et al. Prognosis of breast cancer after supraclavicular lymph node metastasis: not a distant metastasis. Ann Surg Oncol. 2006;13:1457-65.

32. Bagaria SP, Ray PS, Sim MS, Ye X, Sahmonki JM, Cui X, et al. Personalizing breast cancer staging by the inclusion of ER, PR, and HER2. JAMA Surg. 2014;149:125-9.

33. Hwang ES, Lichtensztajn DY, Gomez SL, Fowble B, Clarke CA. Survival after lumpectomy and mastectomy for early stage invasive breast cancer: the effect of age and hormone receptor status. Cancer. 2013;119:1402-11.

34. Park YH, Lee SJ, Cho EY, Choi YL, Lee JE, Nam SJ, et al. Clinical relevance of TNM staging system according to breast cancer subtypes. Ann Oncol. 2011;22:1554-60.

\section{Submit your next manuscript to BioMed Central and we will help you at every step:}

- We accept pre-submission inquiries

- Our selector tool helps you to find the most relevant journal

- We provide round the clock customer support

- Convenient online submission

- Thorough peer review

- Inclusion in PubMed and all major indexing services

- Maximum visibility for your research

Submit your manuscript at www.biomedcentral.com/submit
Biomed Central 